\title{
Catechins and Caffeine Inhibit Fat Accumulation in Mice through the Improvement of Hepatic Lipid Metabolism
}

\author{
Chikako Sugiura, ${ }^{1,2}$ Shiho Nishimatsu, ${ }^{3}$ Tatsuya Moriyama, ${ }^{4}$ Sayaka Ozasa, ${ }^{5}$ \\ Teruo Kawada, ${ }^{5}$ and Kazutoshi Sayama ${ }^{1,3}$ \\ ${ }^{1}$ Department of Bioscience, Graduate School of Science and Technology, Shizuoka University, 836 Ohya, Shizuoka-shi 422-8529, Japan \\ ${ }^{2}$ Department of Health Promotional Sciences, Faculty of Health Promotional Sciences, Hamamatsu University, 1230 Miyakoda-cho, \\ Hamamatsu-shi 431-2102, Japan \\ ${ }^{3}$ Department of Applied Biological Chemistry, Faculty of Agriculture, Shizuoka University, 836 Ohya, Shizuoka-shi 422-8529, Japan \\ ${ }^{4}$ Department of Applied Cell Biology, Graduate School of Agriculture, Kinki University, Nakamachi, Nara 631-8505, Japan \\ ${ }^{5}$ Division of Food Science and Biotechnology, Graduate School of Agriculture, Kyoto University, Kyoto 606-8502, Japan
}

Correspondence should be addressed to Kazutoshi Sayama, acksaya@ipc.shizuoka.ac.jp

Received 10 January 2012; Revised 18 February 2012; Accepted 5 April 2012

Academic Editor: Eric Doucet

Copyright (C) 2012 Chikako Sugiura et al. This is an open access article distributed under the Creative Commons Attribution License, which permits unrestricted use, distribution, and reproduction in any medium, provided the original work is properly cited.

\begin{abstract}
To elucidate the inhibiting mechanisms of fat accumulation by catechins, caffeine, and epigallocatechin gallate (EGCG), ICR mice were fed diets containing either $0.3 \%$ catechins or $0.1 \%$ EGCG and/or $0.05 \%$ caffeine for 4 weeks. After the feeding, intraperitoneal adipose tissues weights were significantly lower in the caffeine, catechins + caffeine, and EGCG + caffeine groups compared to controls. Hepatic fatty acid synthase (FAS) activity in the catechins + caffeine group was significantly lower, and the activities of acyl-CoA oxidase (ACO) and carnitine palmitoyltransferase-II (CPT-II) were significantly higher, compared to the control group. However, these activities were not observed in the other groups. FAS mRNA expression levels in the catechins + caffeine group were significantly lower than in the control group. ACO and CPT-II mRNA levels were not different among all of the treatment groups. These findings indicate that the inhibitory effects of fat accumulation via a combination of catechins, EGCG, or caffeine were stronger collectively than by either catechins, EGCG, or caffeine alone. Moreover, it was demonstrated that the combination of catechins and caffeine induced inhibition of fat accumulation by suppression of fatty acid synthesis and upregulation of the enzymatic activities involved in $\beta$-oxidation of fatty acid in the liver, but this result was not observed by combination of EGCG and caffeine.
\end{abstract}

\section{Introduction}

Recently, metabolic syndrome has become a serious health problem in advanced countries, and emphasis has been placed on reducing obesity, which is believed to be the main cause of metabolic syndrome. There are numerous reports regarding the antiobesity effects of certain food components. In particular, it was found that green tea and its components, catechins, caffeine, and theanine, have suppressive effects on fat accumulation. Sayama et al. reported that an administration of a diet containing $2 \%$ green tea powder suppressed body weight gain and endoceliac fat accumulation and decreased triglycerides (TG) and total cholesterol (TC) in the liver, as well as TG in serum, without reducing food intake in mice [1]. Moreover, to elucidate the inhibitory effects by catechins and caffeine, two major components of green tea, mice were fed diets containing $0.3 \%$ catechins and $0.05 \%$ caffeine, corresponding to the concentrations found in a $2 \%$ green tea powder diet. As a result, it was shown that catechins and caffeine together had similar inhibitory effects of fat accumulation as green tea [2]. Therefore, it was suggested that the combination of catechins and caffeine might be the most effective of all green tea components in suppressing fat accumulation.

It has been reported that catechins, the major component of green tea, have suppressive effects on fat accumulation. 
Body fat gain in mice fed a high-fat diet was significantly suppressed by catechins, and mRNA expressions of acyl-CoA oxidase (ACO) and medium-chain acyl-CoA dehydrogenase (MCAD), which are related to $\beta$-oxidation within the liver, were upregulated $[3,4]$. It was also demonstrated that body weight gain, body fat accumulation, TC and TG levels in the liver, and TG levels in serum were suppressed in rats fed a normal diet and water containing $0.5 \%$ catechins [5]. Additionally, a diet containing $1 \%$ catechins decreased TG levels and the enzymatic activities of fatty acid synthase (FAS), an enzyme involved in fatty-acid synthesis in rat livers [6].

Furthermore, caffeine, another component of green tea, also possesses antiobesity effects. Rats fed a high-fat diet with $0.05 \%$ or $0.1 \%$ caffeine demonstrated a reduction in body fat mass, as well as hepatic and serum TC and TG levels. Moreover, serum catecholamine concentrations were increased in rats that were orally administered $5 \mathrm{mg} / \mathrm{kg}$ of caffeine [7]. An oral administration of $0.04 \%$ caffeine decreased the weight of the parametrial fats pad by $43 \%$ in mice [8]. Furthermore, caffeine intake was found to be associated with a reduction in body fat via catecholamine-induced lipolysis and an increase in lipid consumption via thermogenesis within brown adipose tissues [9]. Additionally, it was demonstrated that the thermogenic actions of caffeine were further enhanced by catechins. However, the mechanisms behind the inhibitory effects of fat accumulation by both catechins and caffeine, in particular, their effect on lipid metabolism within liver, are still unclear.

It has been reported that epigallocatechin gallate (EGCG), which makes up about $30 \%$ of green tea's catechins, also has suppressive effects on fat accumulation. Mice fed a high-fat diet containing EGCG demonstrated a reduction in body fat accumulation and body weight gain [10], an increase in the mRNA expression levels of sterol regulatory element-binding protein-1c (SREBP-1c) and FAS, and a reduction in the mRNA expression levels of carnitine palmitoyltransferase-I (CPT-I) and uncoupling protein 2 (UCP2), all of which are related to $\beta$-oxidation in the adipose tissue of mice [11]. Moreover, when rats were fed a high-fat diet and water containing green tea or EGCG, the gene expression of SREBP-1c, FAS, malonyl CoA decarboxylase (MCD), and acetyl CoA carboxylase (ACC), which are involved in fattyacid synthase, as well as peroxisome proliferator-activated receptor $\alpha(\operatorname{PPAR} \alpha)$, CPT-I, and ACO, which are involved in the $\beta$-oxidation within the liver, were increased by green tea, but not by EGCG [12]. Therefore, it was suggested that EGCG may not affect lipid metabolism in the liver. However, the effects of EGCG and caffeine on lipid metabolism in the liver are yet to be investigated.

The above-mentioned reports demonstrated that catechins, EGCG, and caffeine have suppressive effects on fat accumulation and that the mechanism of the action of catechins may be related to the modification of enzymes involved in lipid metabolism, as well as their transcription factors, within the liver and adipose tissue. We proposed that the combination of catechins and caffeine may have a stronger effect on hepatic lipid metabolism than either alone, since catechins and caffeine appear to be synergistic in their inhibitory effects of fat accumulation.
Thus, to elucidate the mechanism of inhibitory action of fat accumulation by catechins and caffeine, particularly, the effects to hepatic lipid metabolism and to clarify what components of green tea catechins are responsible for the inhibitory effects of fat accumulation by the diet with catechins and caffeine, we investigated the effects of a combination of either catechins or EGCG with caffeine on body and intraperitoneal adipose tissue (IPAT) weights, serum and hepatic lipid levels, as well as the enzymatic activities, and mRNA expression levels of lipid-metabolizing enzymes.

\section{Materials and Methods}

2.1. Animals and Diets. Female 10-week-old ICR mice weighing approximately $30-32 \mathrm{~g}$ were purchased from an animal breeder (Japan SLC Co., Hamamatsu, Japan) and used in this study. In previous reports, ICR mice were used for the research about inhibitory effects of fat accumulation by green tea and green tea components $[1,2]$. Weight of intraperitoneal adipose tissue in the control mice was remarkably increased with age, and the increase ratio became about $9 \%$ of body weight until 5 months old, even by feeding of commercial diet. Therefore, we also used this mouse strain as a model mouse which characterizes severe fat accumulation in vivo. The mice were maintained on standard powdered laboratory chow MF (Oriental Yeast Co. Ltd., Tokyo, Japan).

Catechin powder was purchased from Mitsui Norin Co. (Tokyo, Japan), and EGCG powder was obtained from TEAVIGO, Roche Vitamin Japan Co. (Tokyo, Japan). Caffeine was purchased from WAKO Pure Chemical Industries, Ltd. (Osaka, Japan). Catechin, EGCG, and caffeine powder were mixed into a standard powder diet for mice at concentrations of $0.3 \%, 0.1 \%$, and $0.05 \%$, respectively, alone and in combination. These concentrations corresponded with those found in a $2 \%$ green tea powder diet.

Food intake was measured in a group of 3 mice that were individually housed in mouse cages and fed either a mixed or nonmixed diet and tap water ad libitum for 4 weeks. All mice were kept in an air-conditioned (temperature of $24 \pm 2^{\circ} \mathrm{C}$ and humidity of $50 \pm 10 \%)$ and light-controlled ( $12 \mathrm{~h}$ day: $12 \mathrm{~h}$ night; lights on from 08:00 to 20:00) animal room.

Ethical approval for the study was obtained from the Committee for Animal Experimentation of Shizuoka University.

2.2. Sampling Procedures. After feeding, all mice were fasted for $6 \mathrm{~h}$ prior to sacrifice. Following the fasting period, each mouse was deeply anesthetized by an overdose of diethyl ether, and blood was withdrawn from the heart. Serum was isolated from clotted blood by centrifugation at $800 \times \mathrm{g}$ for $15 \mathrm{~min}$ at $4^{\circ} \mathrm{C}$. Livers and IPAT were weighted. Serum and livers were stored at $-80^{\circ} \mathrm{C}$ until use for the determination of the lipid levels, as well as genetic and enzymatic activities related to lipid metabolism.

2.3. Lipid Analysis. Serum concentrations of TC and TG were measured enzymatically with test kits (cholesterol Ctest and triglyceride G-test, Wako Co., Japan). Lipids within 
the liver were extracted by using the method described by Folch et al. [13]. TC and TG within the extract were analyzed via the methods described by Zak [14] and Fletcher [15], respectively.

2.4. Sample Preparation for Enzymatic Activity Assay and Western Blotting. Frozen liver samples were thawed and homogenized at $4^{\circ} \mathrm{C}$ in a homogenizing solution containing $3 \mathrm{mM}$ Tris- $\mathrm{HCl}$ ( $\mathrm{pH} 7.2$ ), $0.25 \mathrm{M}$ sucrose, $1 \mathrm{mM}$ DTT (dithiothreitol), $25 \mu \mathrm{M}$ ALLN (N-acetyl-leucyl-leucyl-norleucinal), $100 \mu \mathrm{M}$ AEBSF (4-(2-aminoethyl) benzenesulfonyl-fluoride), $100 \mu \mathrm{M}$ leupeptin (acetyl-leucyl-leucyl-argininal), and $10 \mu \mathrm{M}$ E64 (N-(N-(L-3-trans-carboxirane-2carbonyl)-L-leucyl)-agmatine. The resultant supernatant was used in an assay that determined CPT-II involvement in $\beta$-oxidation of fatty acid. The rest of the homogenizing solution was centrifuged at $600 \times \mathrm{g}$ for $10 \mathrm{~min}$ at $4^{\circ} \mathrm{C}$, and some of the supernatant was used for the measurement of ACO activity. The remainder of the supernatant was then centrifuged at $9000 \times \mathrm{g}$ for $10 \mathrm{~min}$ at $4^{\circ} \mathrm{C}$, and a portion of the supernatant was then used for the measurement of FAS activity. Prior to measuring enzymatic activity, protein concentrations were determined with the Assay Dye Concentrate method. The solutions used for CPT-II and ACO activity measurements were diluted to $10 \mathrm{mg} / \mathrm{mL}$ and $15 \mathrm{mg} / \mathrm{mL}$, respectively. The solution used for Western Blotting was diluted to $3 \mu \mathrm{g} / \mu \mathrm{L}$.

2.5. Measurement of FAS, ACO, and CPT-II Activity. The enzymatic activity of FAS was measured using a method described by Kelley et al. [16], Nepokroeff et al. [17], and Moriyama et al. [18]. ACO and CPT-II activities were measured, as previously described by Hashimoto et al. [19], Osumi and Hashimoto [20], Markwell et al. [21], and Moriyama et al. [18]. All enzyme activities were measured via a spectrophotometric assay using a recording spectrophotometer (model V630-BIO, JASCO, Tokyo, Japan).

2.6. Western Blot Analysis. Total protein was extracted, and $30 \mathrm{ug}$ were loaded in each lane. Protein was separated on a $7.5 \%$ SDS-PAGE (sodium dodecyl sulfate-polyacrylamide gel electrophoresis) and transferred onto PVDF membranes using a semidry blotter (ATTO, Tokyo, Japan). Membranes were blocked for $1 \mathrm{~h}$ in Tris-buffered saline (TBS) containing $2 \%$ nonfat dried milk and $0.1 \%$ Tween 20 , and incubation with primary and secondary antibodies in TBS containing $2 \%$ skim milk for $1.5 \mathrm{~h}$ and $1 \mathrm{~h}$, respectively. The membranes were then washed in TBS containing $0.1 \%$ Tween 20 . The primary antibodies used were rabbit antifatty acids synthase polyclonal antibodies, and the secondary antibodies were horseradish peroxidase-conjugated goat anti-rabbit IgG, $\mathrm{F}\left(\mathrm{ab}^{\prime}\right) 2$ (Santa Cruz Biotech). Lastly, each protein band was detected via chemiluminescence (light capture II system, ATTO, Tokyo, Japan). Signal intensities of the PVDF membranes were measured with Image J software (NIH, USA).

2.7. RNA Preparation and Semiquantitative RT-PCR. Livers were dissected from each animal and stored at $-80^{\circ} \mathrm{C}$ in RNAlater solution (Applied Biosystems, NJ, USA) for subsequent RNAs isolation. Total RNAs were isolated with
Isogen (Nippongene, Toyama, Japan), according to the manufacturer's instructions, followed by deoxyribonuclease digestion. The extracted RNA was analyzed by reverse transcription polymerase chain reaction (RT-PCR) using RNA PCR kits (Applied Biosystems, NJ, USA) according to the supplier's instructions, with the exception of RT, which was performed via an oligo-d(T)16. PCR was performed in a 2720 Thermal Cycler (Applied Biosystems) according to the following specifications: (i) $94^{\circ} \mathrm{C}$ for $45 \mathrm{~s}$ to denature the DNA, $60^{\circ} \mathrm{C}$ for $45 \mathrm{~s}$ to allow for primer annealing, and $72^{\circ} \mathrm{C}$ for $80 \mathrm{~s}$ for extension, all of which were performed for 34 cycles (FAS), (ii) $95^{\circ} \mathrm{C}$ for $1 \mathrm{~min}$ to denature the DNA, $62^{\circ} \mathrm{C}$ for $2 \mathrm{~min}$ to allow for primer annealing, and $72^{\circ} \mathrm{C}$ for $1.5 \mathrm{~min}$ for extension, all of which were performed for 32 cycles (ACO), (iii) $94^{\circ} \mathrm{C}$ for $45 \mathrm{~s}$ to denature the DNA, $55^{\circ} \mathrm{C}$ for $45 \mathrm{~s}$ to allow for primer annealing, and $72^{\circ} \mathrm{C}$ for $30 \mathrm{~s}$ for extension, all of which were performed for 26 cycles (CPTII), (iv) $94^{\circ} \mathrm{C}$ for $1 \mathrm{~min}$ to denature the DNA, $55^{\circ} \mathrm{C}$ for $1 \mathrm{~min}$ to allow for primer annealing, and $72^{\circ} \mathrm{C}$ for 1 min for extension, all of which were performed for 32 cycles (SREBP1c), (v) $94^{\circ} \mathrm{C}$ for $1 \mathrm{~min}$ to denature the DNA, $62^{\circ} \mathrm{C}$ for $2 \mathrm{~min}$ to allow for primer annealing, and $72^{\circ} \mathrm{C}$ for $1.5 \mathrm{~min}$ for extension, all of which were performed for 27 cycles $\left(\operatorname{PPAR} \alpha\right.$ ), and (vi) $94^{\circ} \mathrm{C}$ for $1 \mathrm{~min}$ to denature the DNA, $60^{\circ} \mathrm{C}$ for $1 \mathrm{~min}$ to allow for primer annealing, and $72^{\circ} \mathrm{C}$ for $1 \mathrm{~min}$ for extension, all of which were performed for 25 cycles $(\beta$ actin). The primer sets used were as follows: $5^{\prime}$-CAC AGT TAA GAG TTC ATA C-3' $/ 5^{\prime}$-TGG TTG CTG TGC ATG GCT C-3' (426 bp) for FAS [22], 5' -CCA ACA TGA GGA CTA TAA CTT CCT-3/5'-TAC ATA CGT GCC GTC AGG CTT CAC-3' (661 bp) for ACO [23], 5'-CAC AAC ATC CTG TCC ACC AG-3'/5'-CAT TGC AGC CTA TCC AGT CA-3' (124 bp) for CPT-II [24], 5' -GGA TGT CAC ACA ATG CAA TTC GCT-3' $/ 5^{\prime}$-TCA CAG AAC GGC TTC CTC AGG TT-3' (565 bp) for PPAR $\alpha$ [23], 5' -GGA GCC ATG GAT TGC ACA TT-3'/5'-AGG AAG GCT TCC AGA GAG GA-3' (191 bp) for SREBP-1c [25], and 5'-GCC AAC CGT GAA AAG ATG3'/5'-GAA GGA AGG CTG GAA AAG A-3' (461 bp) for $\beta$-actin [26]. The amplified DNA was subjected to electrophoresis on a $1.5 \%$ agarose gel and stained with ethidium bromide. The intensity of each PCR product for each gene of interest was measured with Image J software, and the resulting densitometric value was compared to $\beta$-actin, the internal standard.

2.8. Statistical Analysis. A Dunnett's multiple comparison test was used for statistical comparisons, and differences were considered significant at $P<0.05$.

\section{Results}

3.1. Body, Liver, and Intraperitoneal Adipose Tissue (IPAT) Weights. The body, liver, and IPAT weights of the control and experimental mice are presented in Figure 1. There were no significant differences between the control and any of the catechins, EGCG, and caffeine groups in body and liver weights (Figures 1(a) and 1(b)). IPAT weights were significantly lower in the $0.05 \%$ caffeine and $0.3 \%$ catechins + $0.05 \%$ caffeine groups than the $0.3 \%$ catechins and control 


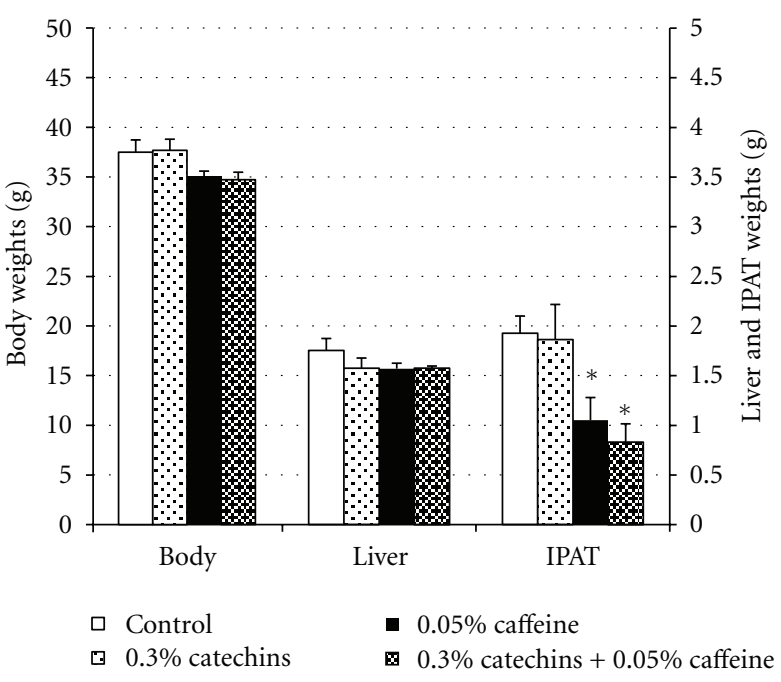

(a)

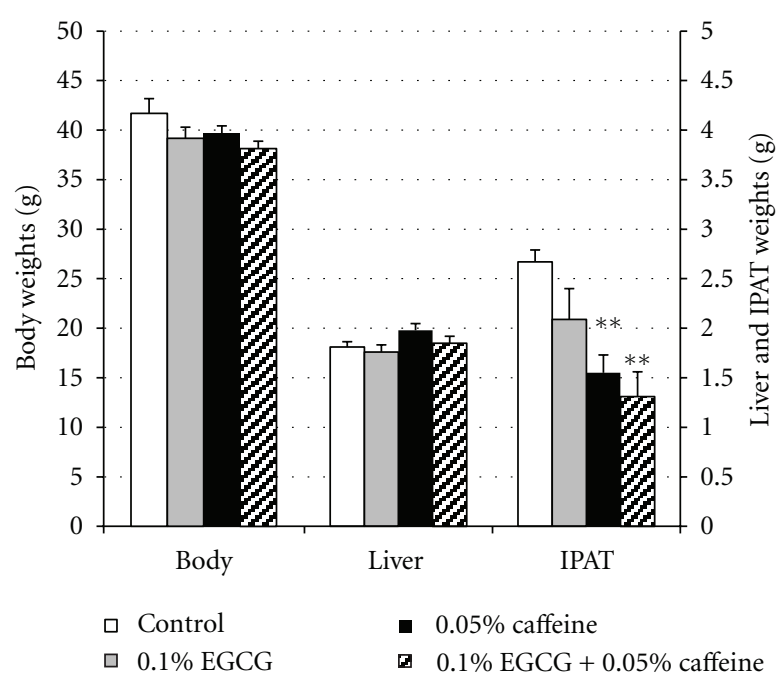

(b)

FIGURE 1: Effects of catechins, EGCG, and caffeine on weights of body, liver, and intraperitoneal adipose tissues in mice. (a) Weights of body, liver, and intraperitoneal adipose tissues (IPAT) in mice fed the diets containing $0.3 \%$ catechins, $0.05 \%$ caffeine, $0.3 \%$ catechins + $0.05 \%$ caffeine for 4 weeks. (b) Weights of body, liver, and IPAT in mice fed the diets containing 0.1\% EGCG, 0.05\% caffeine, $0.1 \%$ EGCG $+0.05 \%$ caffeine for 4 weeks. The means and SE for 10 mice are plotted. ${ }^{*}, * *$ Significant difference compared with the control $(* P<0.05$, ** $P<0.01)$.

groups $(P<0.05)$. The IPAT weights of the $0.05 \%$ caffeine and $0.3 \%$ catechins $+0.05 \%$ caffeine groups were 54.6 and $43.2 \%$, respectively, lower than the control group. Furthermore, IPAT weights were significantly lower in the $0.05 \%$ caffeine and $0.1 \%$ EGCG $+0.05 \%$ caffeine groups than the $0.1 \%$ catechins and control groups $(P<0.01)$. The IPAT weights of the $0.05 \%$ caffeine and $0.1 \%$ EGCG $+0.05 \%$ caffeine groups were 58.2 and $49.1 \%$, respectively, lower than the control group.

3.2. Food Intake. Food intake was compared between the groups which were given catechins, EGCG, and caffeine singly or in combination, and the control group. There were no significant differences between the control group and any of the catechins, EGCG, and caffeine groups (Table 1).

3.3. Lipid Concentrations in Serum and Liver. The serum and hepatic TC and TG levels of all groups are shown in Table 2. There were no significant differences in both TC and TG concentrations within the serum and liver among any of the treatment groups and the control group.

3.4. Enzymatic Activities of Fatty-Acid Synthesis and $\beta$ Oxidation Markers within the Liver. The enzymatic activities of FAS, ACO, and CPT-II within the liver after treatment with catechins, EGCG, and caffeine are presented in Figure 2. FAS activity in the $0.3 \%$ catechins $+0.05 \%$ caffeine group was significantly lower than in the control group $(P<0.05)$. Conversely, the administration of $0.3 \%$ catechins $+0.05 \%$ caffeine significantly increased the activities of both ACO and CPT-II $(P<0.05)$. However, there were no significant effects on the enzymatic activities of FAS, ACO, and CPTII with either $0.3 \%$ catechins or $0.05 \%$ caffeine treatment.
Furthermore, there were no significant differences in all enzyme activities between the 0.1\% EGCG, 0.05\% caffeine, $0.1 \%$ EGCG $+0.05 \%$ caffeine, and control groups.

3.5. FAS Protein Levels in the Liver. Hepatic FAS protein levels in the control and experimental groups are presented in Figure 3. FAS protein levels in the $0.3 \%$ catechins + $0.05 \%$ caffeine group tended to be lower than in the control group $(P=0.053)$ (Figure $3(\mathrm{a}))$. There were no significant differences in FAS protein levels between the other experimental groups and the control group (Figures 3(a) and 3(b)).

3.6. Gene Expression of FAS, PPAR $\alpha$, ACO, CPT-II, and SREBP-1c in Liver. The expression levels of hepatic FAS, ACO, and CPT-II mRNA of all experimental groups are presented in Figure 4 . The expression of FAS mRNA in the $0.3 \%$ catechins $+0.05 \%$ caffeine group was significantly lower than in the control group $(P<0.05)$. However, treatment with either $0.3 \%$ catechins or $0.05 \%$ caffeine did not have an effect on FAS mRNA levels. The expression of ACO and CPT-II mRNA was not significantly different between all treatment groups and the control group. Moreover, the expression levels of PPAR $\alpha$ and SREBP-1c mRNA within the liver was not different in any experimental groups (data not shown).

\section{Discussion}

In the present study, we focused that the inhibitory effects of fat accumulation by catechins, EGCG, and caffeine and the inhibitory mechanisms through lipid metabolism in the liver. As a result, it was demonstrated that the suppressive effects on fat accumulation by a combination of catechins and caffeine, or EGCG and caffeine, were stronger than a single 
TABLE 1: Effects of catechins, EGCG, and caffeine on food intake in mice.

\begin{tabular}{lccc}
\hline \multicolumn{4}{c}{$(\mathrm{A})$} \\
\hline \multicolumn{4}{c}{ Dietary groups } \\
Control & $0.3 \%$ catechins & $0.05 \%$ caffeine & $0.3 \%$ catechins $+0.05 \%$ caffeine \\
$138.15 \pm 3.39$ & $134.61 \pm 3.71$ & $139.62 \pm 2.58$ & $138.68 \pm 2.71$ \\
\hline \multicolumn{4}{c}{ (B) } \\
\hline \multicolumn{4}{c}{ Dietary groups } \\
Control & $0.1 \%$ EGCG & $0.05 \%$ caffeine & $0.1 \%$ EGCG $+0.05 \%$ caffeine \\
$137.93 \pm 3.48$ & $129.97 \pm 2.40$ & $140.90 \pm 3.89$ & $136.82 \pm 3.95$ \\
\hline
\end{tabular}

Values $(g)$ are means of total food intake during 4 weeks per mouse \pm SE for 3 mice.

TABLE 2: Effects of catechins, EGCG, and caffeine on lipid levels in serum and liver in mice.

\begin{tabular}{|c|c|c|c|c|}
\hline \multicolumn{5}{|c|}{ (A) } \\
\hline & \multicolumn{4}{|c|}{ Dietary groups } \\
\hline & Control & $0.3 \%$ catechins & $0.05 \%$ caffeine & $0.3 \%$ catechins $+0.05 \%$ caffeine \\
\hline \multicolumn{5}{|l|}{ Serum lipids } \\
\hline Triglycerides $(\mathrm{mg} / \mathrm{mL})$ & $1.39 \pm 0.26$ & $1.41 \pm 0.23$ & $1.29 \pm 0.18$ & $1.14 \pm 0.19$ \\
\hline Total cholesterol (mg/mL) & $1.39 \pm 0.17$ & $1.34 \pm 0.08$ & $1.28 \pm 0.10$ & $1.34 \pm 0.12$ \\
\hline \multicolumn{5}{|l|}{ Liver lipids } \\
\hline Triglycerides (mg/g) & $78.11 \pm 6.21$ & $74.65 \pm 7.97$ & $77.98 \pm 9.64$ & $73.98 \pm 6.57$ \\
\hline Total cholesterol (mg/g) & $28.74 \pm 1.86$ & $27.15 \pm 0.40$ & $30.18 \pm 3.21$ & $26.04 \pm 1.14$ \\
\hline \multicolumn{5}{|c|}{ (B) } \\
\hline & \multicolumn{4}{|c|}{ Dietary groups } \\
\hline & Control & $0.1 \%$ EGCG & $0.05 \%$ caffeine & $0.1 \%$ EGCG $+0.05 \%$ caffeine \\
\hline \multicolumn{5}{|l|}{ Serum lipids } \\
\hline Triglycerides $(\mathrm{mg} / \mathrm{mL})$ & $1.56 \pm 0.25$ & $1.34 \pm 0.11$ & $1.53 \pm 0.09$ & $1.50 \pm 0.10$ \\
\hline Total cholesterol (mg/mL) & $1.21 \pm 0.10$ & $1.08 \pm 0.07$ & $1.29 \pm 0.14$ & $1.15 \pm 0.04$ \\
\hline \multicolumn{5}{|l|}{ Liver lipids } \\
\hline Triglycerides (mg/g) & $103.86 \pm 20.21$ & $59.74 \pm 11.43$ & $83.70 \pm 16.65$ & $80.67 \pm 9.90$ \\
\hline Total cholesterol (mg/g) & $29.08 \pm 1.59$ & $26.06 \pm 2.01$ & $26.30 \pm 1.91$ & $27.66 \pm 0.87$ \\
\hline
\end{tabular}

Values are means \pm SE for 10 mice.

administration of either catechins, EGCG, or caffeine in mice. Moreover, it was revealed that the inhibitory action of the catechins and caffeine combination appears to be related to an improvement in hepatic lipid metabolism.

Zheng et al. previously reported that mice fed a diet containing $0.3 \%$ catechins and $0.05 \%$ caffeine in combination for 16 weeks demonstrated a significant suppression in both body weight gain and endoceliac fat accumulation compared to mice fed $0.3 \%$ catechins or $0.05 \%$ caffeine alone [2]. These findings are corroborated by our observations, where IPAT weights, but not body weights, were significantly reduced by a 4-week diet containing the same concentrations of catechins and caffeine. Therefore, it appears that a combination of catechins and caffeine possesses very strong suppressive effects on fat accumulation, even after a short-term administration period.

It has been previously shown that a 1-month high-fat diet together with $0.5 \%$ catechins induces significant reductions in visceral fat weight [27]. Although IPAT weight was not affected by a $0.3 \%$ catechins diet alone (Figure 1), we found that fat accumulation was suppressed by the addition of caffeine. These findings indicate that a lower concentration of catechins is more effective in suppressing fat accumulation in combination with caffeine. Furthermore, there was no suppression of fat accumulation in mice fed diets containing $0.1 \%$ EGCG, an amount that is similar to that found in a $0.3 \%$ catechins diet. It was previously reported that fat accumulation in mice is suppressed by 5- and 10 -fold with diets containing $0.5 \%$ and $1 \%$ EGCG, respectively $[10,11]$. However, IPAT weight in mice fed a diet containing a combination of $0.1 \%$ EGCG and $0.05 \%$ caffeine decreased significantly compared to controls (Figure 1). These results indicate that even low concentrations of EGCG have stronger suppressing effects on fat accumulation in combination with caffeine. Thus, our results clearly demonstrate that the suppression of fat accumulation by catechins or EGCG is augmented in combination with caffeine, which is also a component of green tea. Additionally, EGCG may be an important factor in the inhibitory effects of fat accumulation by catechins and caffeine. However, the inhibitory effects by EGCG and caffeine were lower in comparison to the combination of catechins and caffeine. Therefore, other 


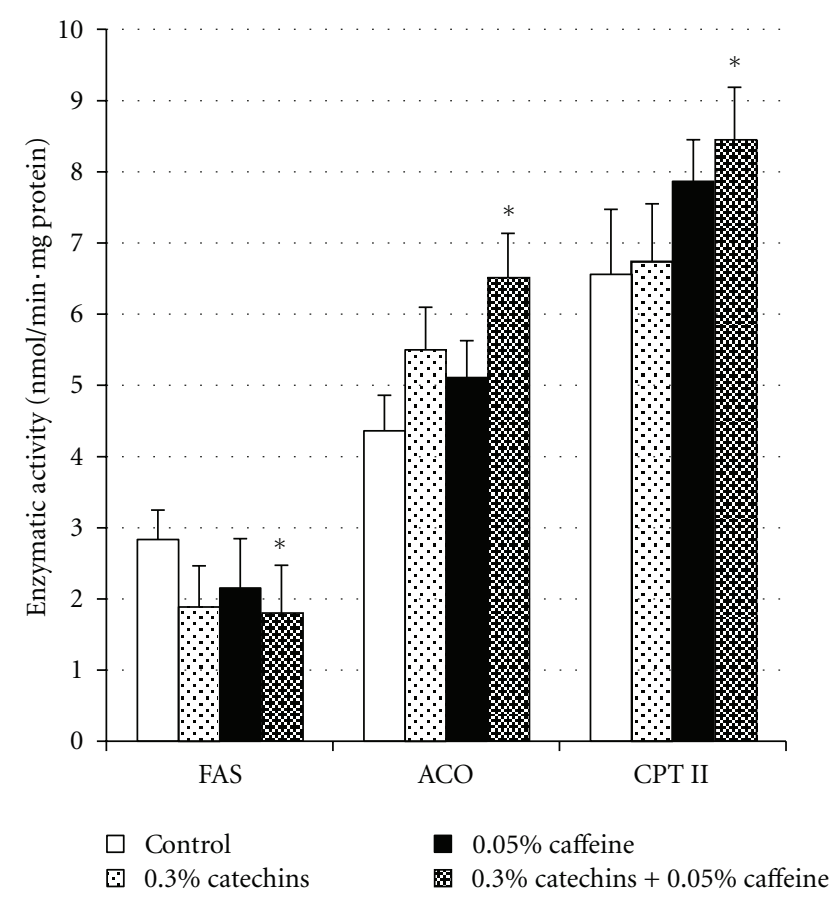

(a)

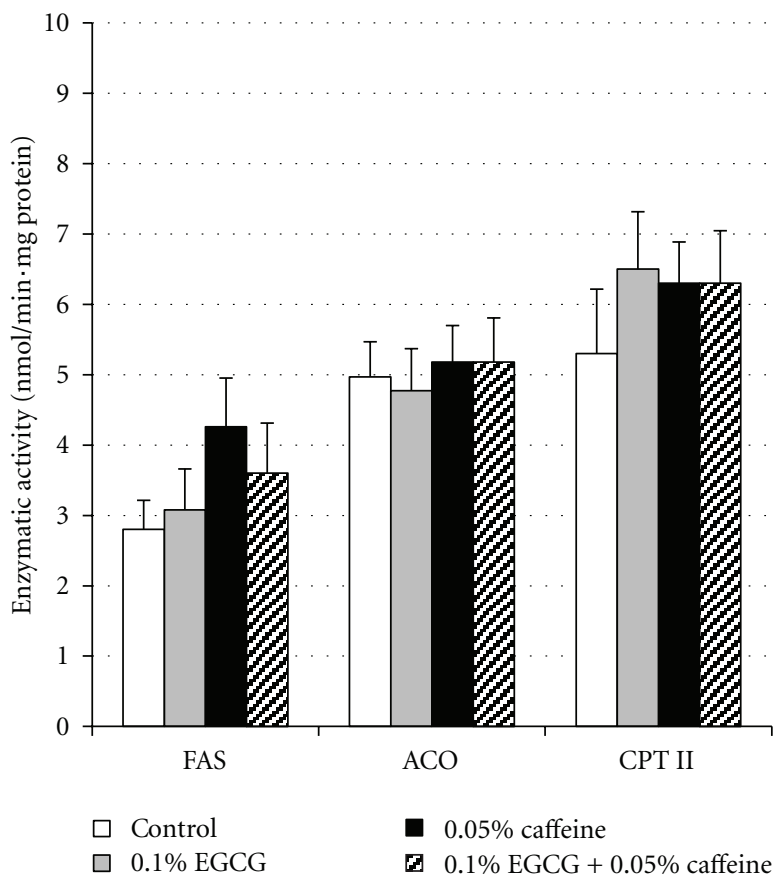

(b)

FIGURE 2: Effects of catechins, EGCG, and caffeine on enzymatic activity related to lipid metabolism in liver in mice. (a) Enzymatic activity of FAS, ACO, and CPT II in liver in mice fed the diets containing $0.3 \%$ catechins, $0.05 \%$ caffeine, $0.3 \%$ catechins $+0.05 \%$ caffeine for 4 weeks. (b) Enzymatic activity of FAS, ACO, and CPT II in liver in mice fed the diets containing 0.1\% EGCG, 0.05\% caffeine, $0.1 \%$ EGCG + $0.05 \%$ caffeine for 4 weeks. FAS: fatty acid synthase, ACO: acyl-CoA oxidase, CPT II: carnitine palmitoyl transferase II. The means and SE for 10 mice are plotted. * Significant difference compared with the control $(P<0.05)$.

catechins, or all catechins including EGCG, may also be involved in suppression of fat accumulation by the combination of catechins and caffeine.

The liver plays an important role in lipid metabolism via various mechanisms, such as fatty-acid synthesis and $\beta$-oxidation. Murase et al. $[3,4]$ reported that the mRNA expression of ACO and MCAD, both enzymes involved in $\beta$ oxidation within the peroxisome and mitochondria of cell, were activated in the livers of mice following an administration of a diet containing $0.5 \%$ catechins. Accordingly, it was proposed that catechins induce their suppressive effects by upregulating the activities of enzymes involved in the $\beta$-oxidation of fatty acid and suppression of enzymatic activities involved in fatty acid synthesis within the liver. However, in another study, a 1\% catechins diet inhibited FAS activity but did not affect the activities of CPT and ACO [6]. In the present study, we found that hepatic ACO and CPTII activities were unchanged by $0.3 \%$ catechins or caffeine alone. However, a combination of $0.3 \%$ catechins and $0.05 \%$ caffeine resulted in an upregulation of these enzymes. These results indicate that caffeine possesses synergistic actions with catechins that act to stimulate $\beta$-oxidation. Interestingly, the gene expression levels of ACO and CPTII were not different in the livers of mice fed a combination of $0.3 \%$ catechins and $0.05 \%$ caffeine. Therefore, it appears that the upregulation of enzymatic activities induced by both catechins and caffeine was stimulated by mechanisms other than those involving an upregulation of gene expression.

One potential mechanism for the suppression of fatty acid synthesis in the liver induced by catechins and caffeine may be related to their effects on the mRNA expression of FAS and subsequent protein synthesis. This is based on the fact that only the $0.3 \%$ catechins and $0.05 \%$ caffeine group demonstrated a downregulation of enzymatic activity and gene expression of FAS, as well as a tendency to have reduced FAS protein levels (Figures 2, 3, and 4). It was reported that a high dose of EGCG (i.e., $0.5 \%$ or more) affects certain enzymes involved in lipid metabolism, including FAS and ACO in the liver $[11,12,28]$. Even though the diet containing $0.1 \%$ EGCG $+0.05 \%$ caffeine suppressed fat accumulation in mice, there were no significant differences in the enzymatic activities and mRNA expression of FAS, ACO, and CPTII (Figures 2 and 4). Thus, it appears that EGCG affects lipid metabolism in adipose tissues rather than in the liver, when its concentration is low or when other catechins are not present. Furthermore, EGCG may play an important role in suppressing fat accumulation and improving lipid metabolism within the liver with catechins and caffeine.

It is known that suppression of fatty acid and TG levels is caused by activation of PPAR $\alpha$ which is a transcription factor involved in the gene expression of fatty acid oxidation enzymes and suppression of SREBP-1c which is transcription 


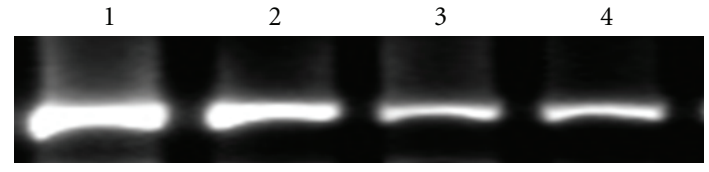

(a1)

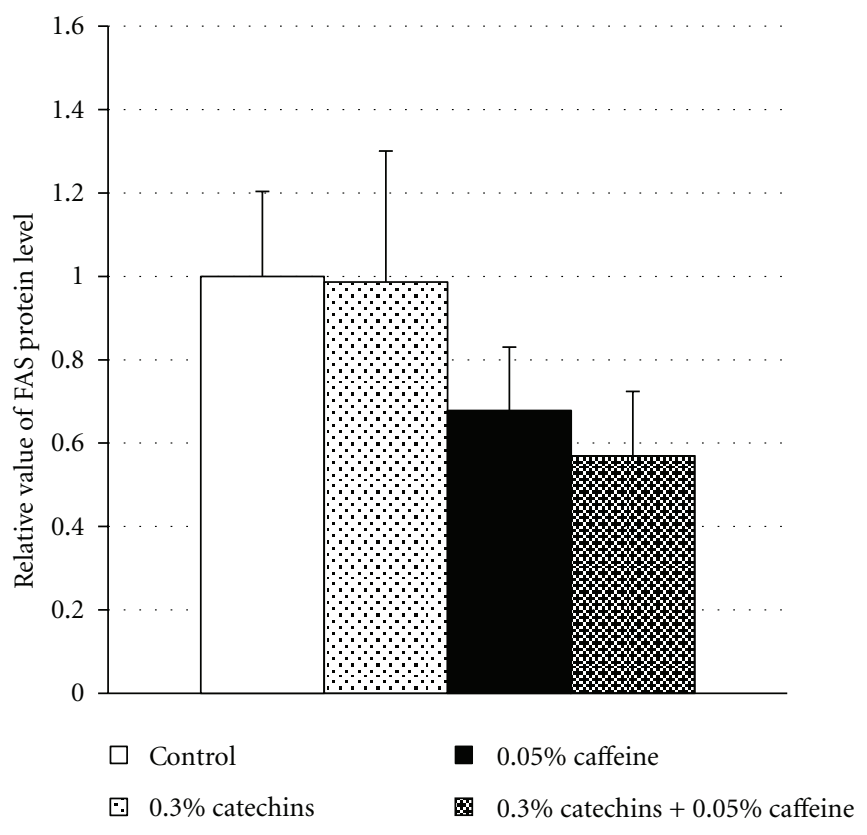

(a2)

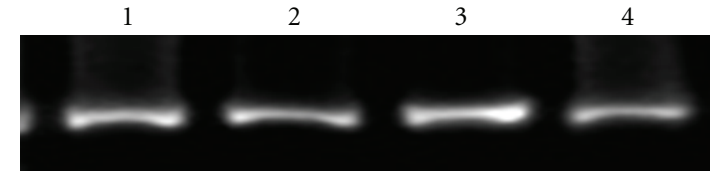

(b1)

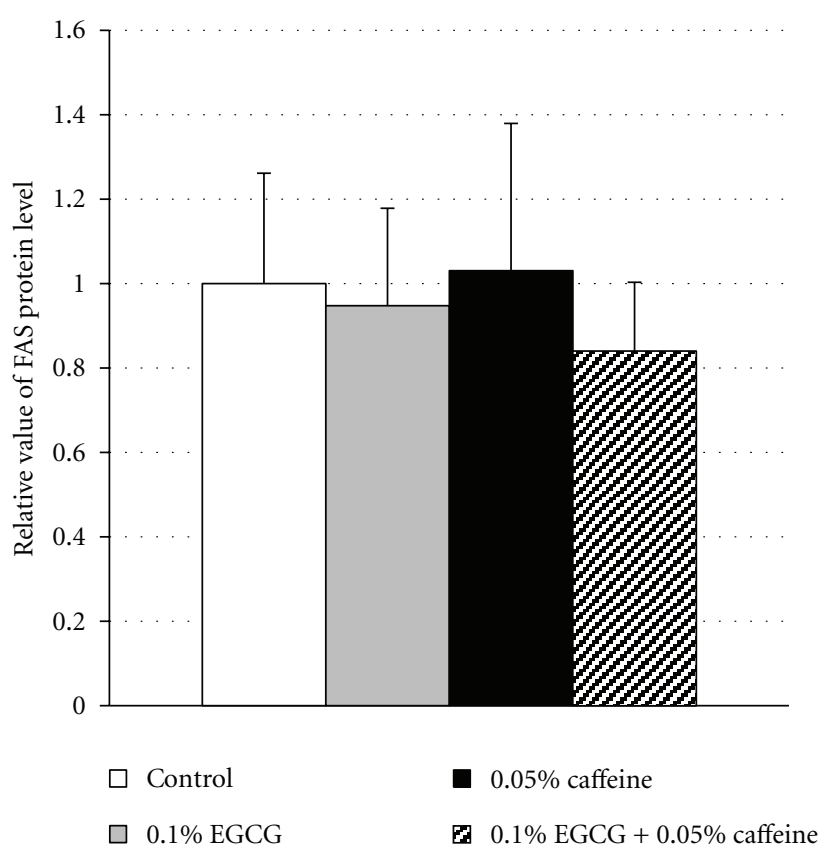

(b2)

FIGURE 3: Effects of catechins, EGCG, and caffeine on protein levels of fatty acid synthase in liver in mice. (a1) Fluorescence intensity of fatty acid synthase (FAS) by Western Blotting analysis in liver in mice fed the diets containing $0.3 \%$ catechins, $0.05 \%$ caffeine, $0.3 \%$ catechins + 0.05\% caffeine for 4 weeks. Lane 1: control, lane 2: $0.3 \%$ catechins, lane 3: $0.05 \%$ caffeine, lane $4: 0.3 \%$ catechins $+0.05 \%$ caffeine. (b1) Fluorescence intensity of FAS by Western Blotting analysis in liver in mice fed the diets containing 0.1\% EGCG, $0.05 \%$ caffeine, $0.1 \%$ EGCG $+0.05 \%$ caffeine for 4 weeks. Lane 1: control, lane 2: 0.1\% EGCG, lane 3: 0.05\% caffeine, lane 4: 0.1\% EGCG + 0.05\% caffeine. (a2 and b2) The protein levels of FAS determined by Western Blotting analysis in liver in mice fed the diets containing catechins and caffeine (a2) and EGCG and caffeine (b2) for 4 weeks. The level was determined from the fluorescence intensity by a image analysis system and showed as a relative value to the control. Results are given as mean \pm SE of 8 mice in each groups.

factor related to gene expression of FAS $[29,30]$. It was reported that there was an increase in the gene expression of PPAR $\alpha, \mathrm{CPT}-1$, and ACO, and a reduction in the gene expression of SREBP-1c, FAS, MCD, and ACC in the livers of rats administered $1 \%$ green tea for 6 months but not in rats that consumed EGCG alone (i.e., $1 \mathrm{mg} / \mathrm{kg} /$ day) [12]. Abe et al. reported that rats administered a green tea beverage containing $540 \mathrm{mg}$ of catechins and $80 \mathrm{mg}$ of caffeine per $350 \mathrm{~mL}$ for 4 months demonstrated an increase in PPAR $\alpha$ gene expression and an inhibition in glucose6-phosphatase and FAS gene expression [31]. However, in our results, the mRNA expressions of PPAR $\alpha$ and SREBP$1 \mathrm{c}$ in liver were not influenced by a diet containing $0.3 \%$ catechins $+0.05 \%$ caffeine and $0.1 \%$ EGCG $+0.05 \%$ caffeine. Therefore, it appears that gene expressions of the enzymes involved in fatty acid metabolism were not affected by a low concentration of catechins and caffeine, and EGCG and caffeine.
In conclusion, it was demonstrated that the combination of catechins and caffeine induced its inhibitory effects of fat accumulation by upregulating the activities of enzymes involved in hepatic $\beta$-oxidation of fatty acid and suppressing the activities of enzymes involved in the fatty acid synthesis, but this was not observed with the EGCG and caffeine combination. Therefore, our results indicate that all catechins, including EGCG or other components, such as epicatechin (EC), epigallocatechin (EGC), and epicatechin gallate (ECG), are involved in suppressing fat accumulation in combination with caffeine.

In this study, we used ICR mouse as a model mouse for severe fat accumulation. Therefore, we did not use a high-fat (calorie) diet. However, our results predicted that a combinational administration of catechins and caffeine or EGCG and caffeine might have antiobesity action. Thus, it was considered that we might be able to examine more elaborate studies of the antiobesity effects by using high-fat 

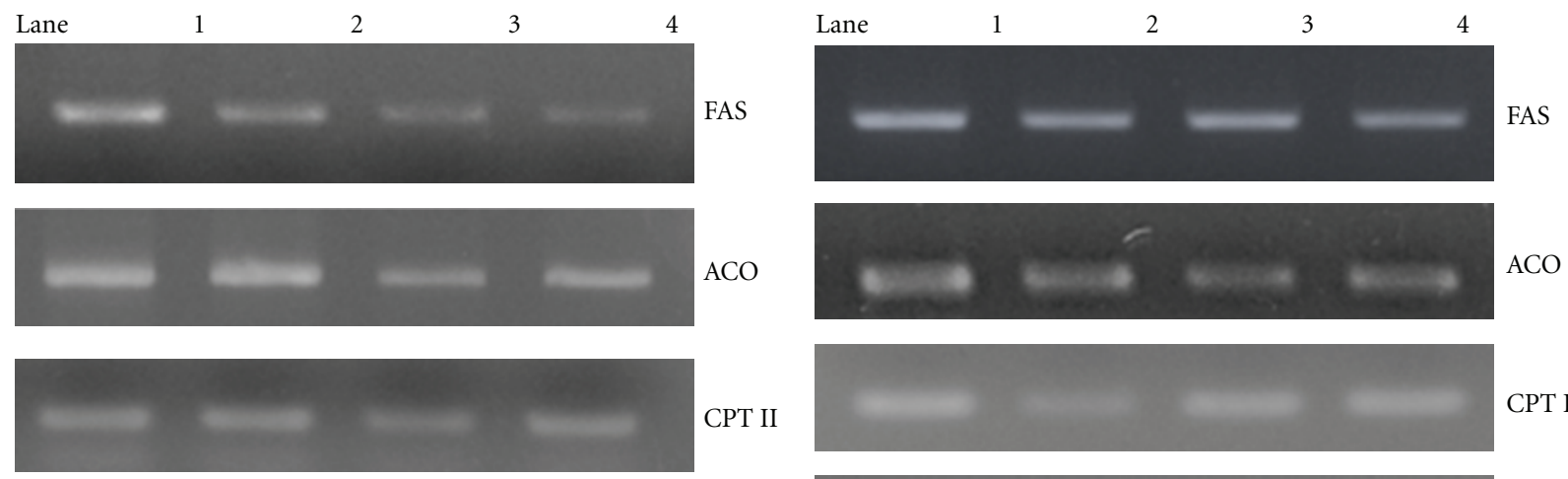

CPT II
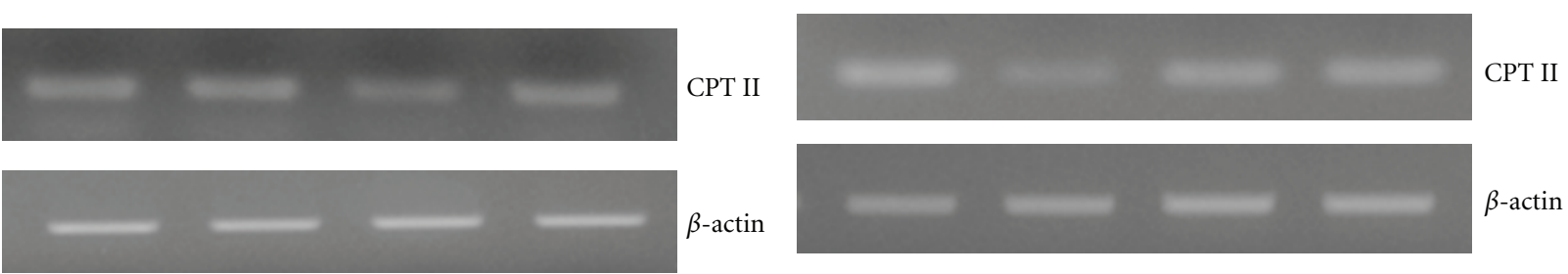

(a1)

(b1)

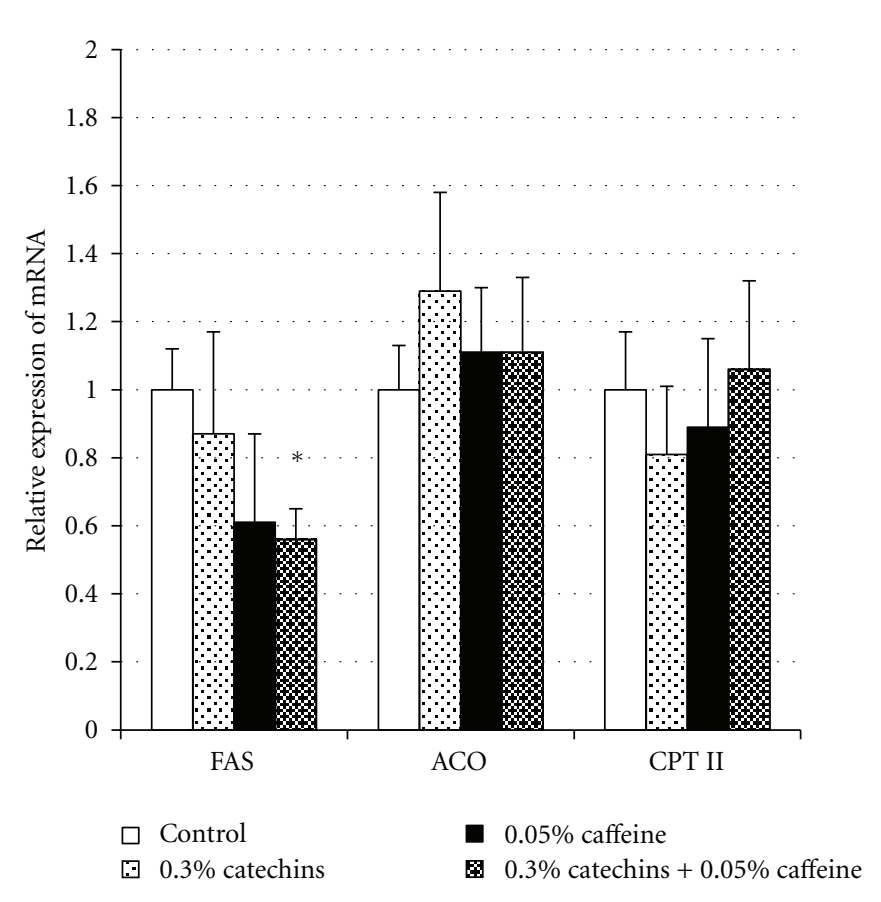

(a2)

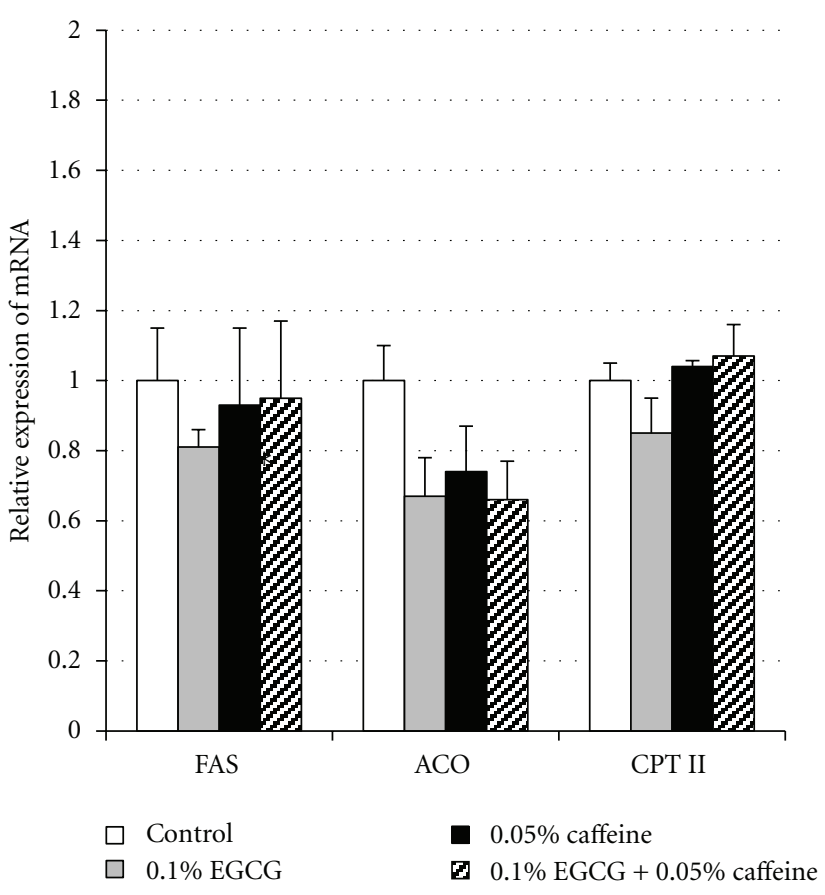

(b2)

FIGURE 4: Effects of catechins, EGCG, and caffeine on the mRNA expression of FAS, ACO, and CPT II in liver in mice. (a1) Fluorescence intensity of mRNA of FAS, ACO, and CPT II by RT-PCR analysis in liver in mice fed the diets containing $0.3 \%$ catechins, $0.05 \%$ caffeine, $0.3 \%$ catechins $+0.05 \%$ caffeine for 4 weeks. Lane 1: control, lane 2: $0.3 \%$ catechins, lane 3: $0.05 \%$ caffeine, lane $4: 0.3 \%$ catechins $+0.05 \%$ caffeine. (b1) Fluorescence intensity of mRNA of FAS, ACO, and CPT II by RT-PCR analysis in liver in mice fed the diets containing $0.1 \%$ EGCG, $0.05 \%$ caffeine, $0.1 \%$ EGCG + 0.05\% caffeine for 4 weeks. Lane 1: control, lane 2: 0.1\% EGCG, lane 3: 0.05\% caffeine, lane 4: 0.1\% EGCG $+0.05 \%$ caffeine. (a2 and b2) Expression levels of mRNA of FAS, ACO, and CPT II determined by RT-PCR analysis in liver in mice fed the diets containing catechins and caffeine (a2) and EGCG and caffeine (b2) for 4 weeks. The level was determined from the fluorescence intensity by a image analysis system and showed as a relative value to the control. Results are given as mean \pm SE of 8 mice in each groups. * Significant difference compared with the control $(P<0.05)$.

diet and other obesity model mice such as C57BL and $o b / o b$ mice. Moreover, we focused on the effects of catechins and caffeine on hepatic function, particularly the lipid metabolism. However, adipose tissues were also very important organs on lipid metabolism in vivo. Thus, we are already proceeding with research about the effects of catechins, EGCG, and caffeine on lipid metabolism in adipose tissues in vivo and adipose cells in vitro. 


\section{References}

[1] K. Sayama, S. Lin, G. Zheng, and I. Oguni, "Effects of green tea on growth, food utilization and lipid metabolism in mice," In Vivo, vol. 14, no. 4, pp. 481-484, 2000.

[2] G. Zheng, K. Sayama, T. Okubo, L. R. Juneja, and I. Oguni, "Anti-obesity effects of three major components of green tea, catechins, caffeine and theanine, in mice," In Vivo, vol. 18, no. 1, pp. 55-62, 2004.

[3] T. Murase, A. Nagasawa, T. Hase, I. Tokimitsu, H. Shimasaki, and H. Itakura, "Dietary tea catechins reduce development of obesity accompanied with gene expression of lipid-metabolizing enzymes in mice," Journal of Oleo Science, vol. 50, no. 9, pp. 711-715, 2001.

[4] T. Murase, A. Nagasawa, J. Suzuki, T. Hase, and I. Tokimitsu, "Beneficial effects of tea catechins on diet-induced obesity: stimulation of lipid catabolism in the liver," International Journal of Obesity, vol. 26, no. 11, pp. 1459-1464, 2002.

[5] Y. Ito, T. Ichikawa, Y. Morohoshi, T. Nakamura, Y. Saegusa, and K. Ishihara, "Effect of tea catechins on body fat accumulation in rats fed a normal diet," Biomedical Research, vol. 29, no. 1, pp. 27-32, 2008.

[6] I. Ikeda, R. Hamamoto, K. Uzu et al., "Dietary gallate esters of tea catechins reduce deposition of visceral fat, hepatic triacylglycerol, and activities of hepatic enzymes related to fatty acid synthesis in rats," Bioscience, Biotechnology and Biochemistry, vol. 69, no. 5, pp. 1049-1053, 2005.

[7] K. Kobayashi-Hattori, A. Mogi, Y. Matsumoto, and T. Takita, "Effect of caffeine on the body fat and lipid metabolism of rats fed on a high-fat diet," Bioscience, Biotechnology and Biochemistry, vol. 69, no. 11, pp. 2219-2223, 2005.

[8] L. Michna, Y. P. Lu, Y. R. Lou, G. C. Wagner, and A. H. Conney, "Stimulatory effect of oral administration of green tea and caffeine on locomotor activity in SKH-1 mice," Life Sciences, vol. 73, no. 11, pp. 1383-1392, 2003.

[9] A. G. Dulloo, C. A. Geissler, A. Collins, and D. S. Miller, "Normal caffeine consumption: influence on thermogenesis and daily energy expenditure in lean and postobese human volunteers," American Journal of Clinical Nutrition, vol. 49, no. 1, pp. 44-50, 1989.

[10] S. Klaus, S. Pültz, C. Thöne-Reineke, and S. Wolfram, "Epigallocatechin gallate attenuates diet-induced obesity in mice by decreasing energy absorption and increasing fat oxidation," International Journal of Obesity, vol. 29, no. 6, pp. 615-623, 2005.

[11] M. S. Lee, C. T. Kim, and Y. Kim, "Green tea (-)-epigallocatechin-3-gallate reduces body weight with regulation of multiple genes expression in adipose tissue of diet-induced obese mice," Annals of Nutrition and Metabolism, vol. 54, no. 2, pp. 151-157, 2009.

[12] N. Chen, R. Bezzina, E. Hinch et al., "Green tea, black tea, and epigallocatechin modify body composition, improve glucose tolerance, and differentially alter metabolic gene expression in rats fed a high-fat diet," Nutrition Research, vol. 29, no. 11, pp. 784-793, 2009.

[13] J. Folch, M. Lees, and G. H. Sloane Stanley, "A simple method for the isolation and purification of total lipides from animal tissues," The Journal of Biological Chemistry, vol. 226, no. 1, pp. 497-509, 1957.

[14] B. Zak, "Simple rapid microtechnic for serum total cholesterol," American Journal of Clinical Pathology, vol. 27, no. 5, pp. 583-588, 1957.
[15] M. J. Fletcher, "A colorimetric method for estimating serum triglycerides," Clinica Chimica Acta, vol. 22, no. 3, pp. 393397, 1968.

[16] D. S. Kelley, G. J. Nelson, and J. E. Hunt, "Effect of prior nutritional status on the activity of lipogenic enzymes in primary monolayer cultures of rat hepatocytes," Biochemical Journal, vol. 235, no. 1, pp. 87-90, 1986.

[17] C. M. Nepokroeff, M. R. Lakshmanan, and J. W. Porter, "Fatty acid synthase from rat liver," Methods in Enzymology, vol. 35, pp. 37-44, 1975.

[18] T. Moriyama, K. Kishimoto, K. Nagai et al., "Soybean $\beta$ conglycinin diet suppresses serum triglyceride levels in normal and genetically obese mice by induction of $\beta$-oxidation, downregulation of fatty acid synthase, and inhibition of triglyceride absorption," Bioscience, Biotechnology and Biochemistry, vol. 68, no. 2, pp. 352-359, 2004.

[19] T. Hashimoto, S. Miyazawa, D. Gunarso, and S. Furuta, “ $\alpha$ amanitin inhibits the oxidation of long chain fatty acids in mouse liver," Journal of Biochemistry, vol. 90, no. 2, pp. 415$421,1981$.

[20] T. Osumi and T. Hashimoto, "Acyl-CoA oxidase of rat liver: a new enzyme for fatty acid oxidation," Biochemical and Biophysical Research Communications, vol. 83, no. 2, pp. 479-485, 1978.

[21] M. A. K. Markwell, E. J. McGroarty, L. L. Bieber, and N. E. Tolbert, "The subcellular distribution of carnitine acyltransferases in mammalian liver and kidney. A new peroxisomal enzyme," Journal of Biological Chemistry, vol. 248, no. 10, pp. 3426-3432, 1973.

[22] D. Liu, D. Pavlovic, M. C. Chen, M. Flodström, S. Sandler, and D. L. Eizirik, "Cytokines induce apoptosis in $\beta$-cells isolated from mice lacking the inducible isoform of nitric oxide synthase (iNOS(-/-))," Diabetes, vol. 49, no. 7, pp. 1116$1122,2000$.

[23] T. Murase, T. Mizuno, T. Omachi et al., "Dietary diacylglycerol suppresses high fat and high sucrose diet-induced body fat accumulation in C57BL/6J mice," Journal of Lipid Research, vol. 42, no. 3, pp. 372-378, 2001.

[24] K. Y. Lee, S. J. Kim, Y. S. Cha et al., "Effect of exercise on hepatic gene expression in an obese mouse model using cDNA microarrays," Obesity, vol. 14, no. 8, pp. 1294-1302, 2006.

[25] T. Kakuma, Y. Lee, M. Higa et al., "Leptin, troglitazone, and the expression of sterol regulatory element binding proteins in liver and pancreatic islets," Proceedings of the National Academy of Sciences of the United States of America, vol. 97, no. 15, pp. 8536-8541, 2000.

[26] Y. Koyama, K. Abe, Y. Sano et al., "Effects of green tea on gene expression of hepatic gluconeogenic enzymes in vivo," Planta Medica, vol. 70, no. 11, pp. 1100-1102, 2004.

[27] S. Meguro, T. Mizuno, K. Onizawa et al., "Effects of tea catechins on diet-induced obesity in mice," Journal of Oleo Science, vol. 50, no. 7, pp. 593-598, 2001.

[28] M. Friedrich, K. J. Petzke, D. Raederstorff, S. Wolfram, and S. Klaus, "Acute effects of epigallocatechin gallate from green tea on oxidation and tissue incorporation of dietary lipids in mice fed a high-fat diet," International Journal of Obesity, vol. 36, no. 5, pp. 735-743, 2012.

[29] S. H. Wong, P. J. Nestel, and R. P. Trimble, "The adaptive effects of dietary fish and safflower oil on lipid and lipoprotein metabolism in perfused rat liver," Biochimica et Biophysica Acta, vol. 792, no. 2, pp. 103-109, 1984.

[30] T. Nakatani, H. J. Kim, Y. Kaburagi, K. Yasuda, and O. Ezaki, "A low fish oil inhibits SREBP-1 proteolytic cascade, while 
a high-fish-oil feeding decreases SREBP-1 mRNA in mice liver: relationship to anti-obesity," Journal of Lipid Research, vol. 44, no. 2, pp. 369-379, 2003.

[31] K. Abe, N. Okada, H. Tanabe et al., "Effects of chronic ingestion of catechin-rich green tea on hepatic gene expression of gluconeogenic enzymes in rats," Biomedical Research, vol. 30, no. 1, pp. 25-29, 2009. 


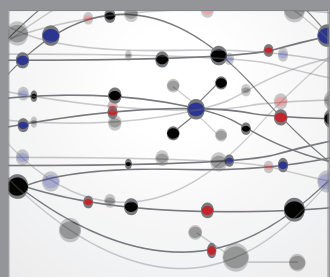

The Scientific World Journal
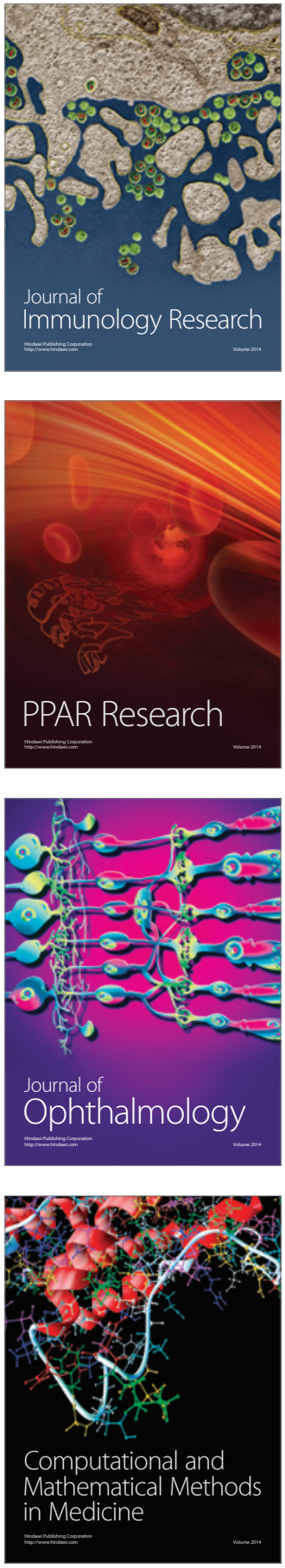

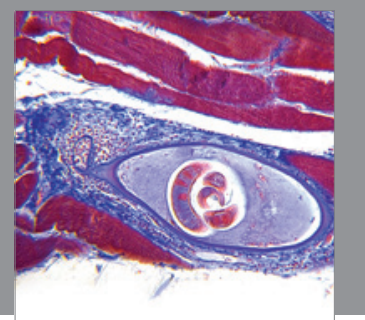

Gastroenterology

Research and Practice
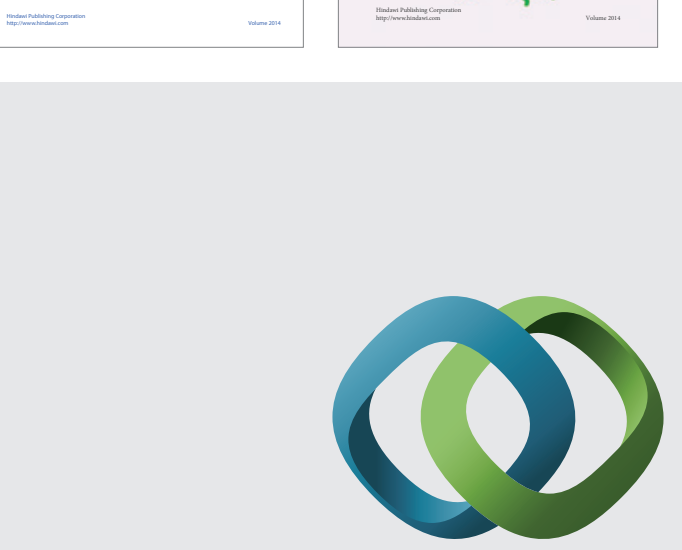

\section{Hindawi}

Submit your manuscripts at

http://www.hindawi.com
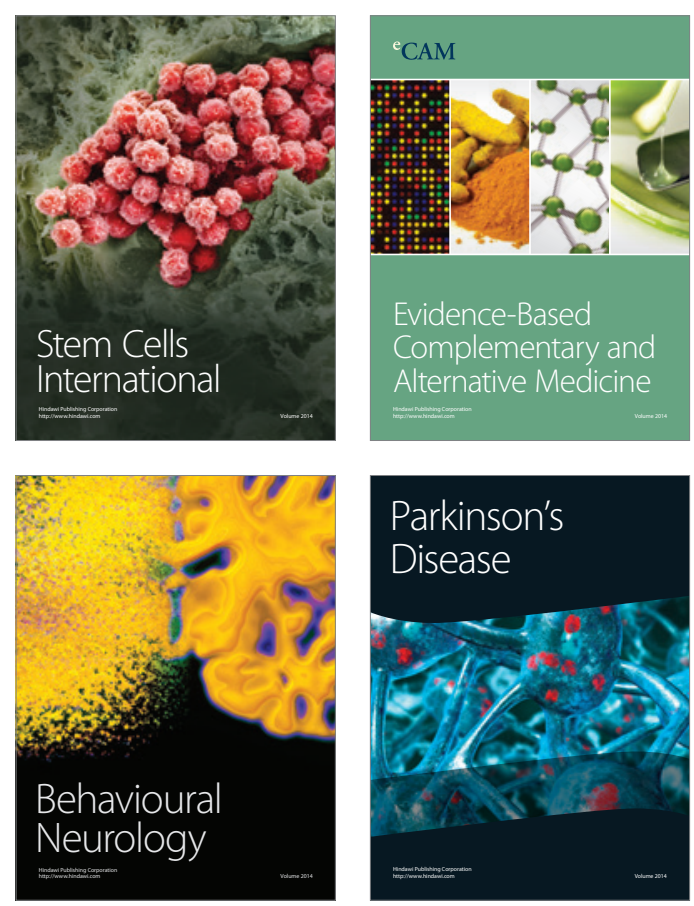

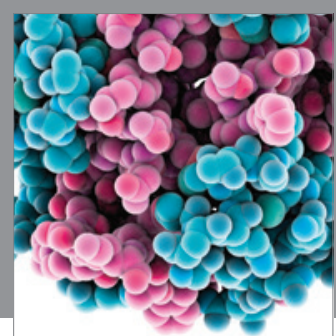

Journal of
Diabetes Research

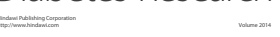

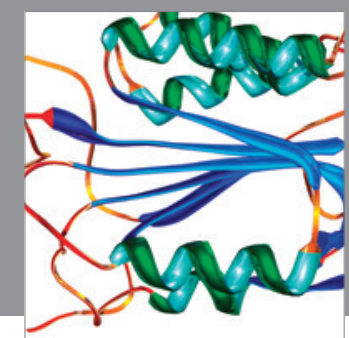

Disease Markers
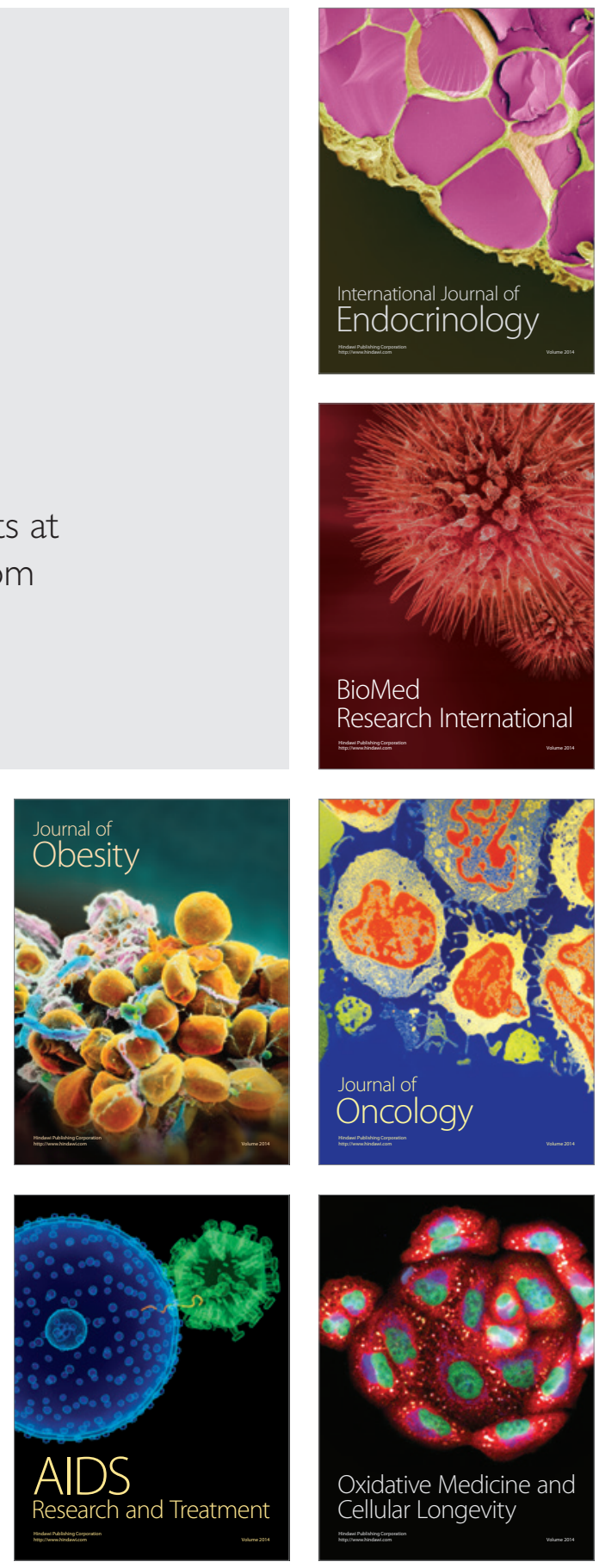